\title{
Creation of line defects in holographic photonic crystals by a double-exposure thresholding method
}

\author{
Chen Chang, Tzu-Min Yan, and Hua-Kuang Liu
}

\begin{abstract}
Recording of periodic variations of amplitude and phase by the interference of coherent laser beams in a hologram offers a natural means for creating one-, two-, and three-dimensional photonic crystals. For device applications such as waveguides in optical communications, one usually needs to create defects in photonic crystals. We present an analysis and an experimental demonstration of a double-exposure method for creating photonic crystals with line defects. The idea is based on the principle of superposition of holographic grating patterns of different spatial periods while the recording medium is held stationary and on the application of a threshold to the recording medium. We use the same symmetrical optical architecture to achieve nondefective and defective holographic photonic crystals. The technique may be extended to the creation of defects based on functional synthesis by means of Fourier series, by use of light sources of other wavelengths with an appropriate high-contrast recording material. (c) 2005 Optical Society of America
\end{abstract}

OCIS codes: $\quad 220.4000,220.4610,090.2880,090.7330$.

\section{Introduction}

The dielectric constant in a photonic crystal may vary in one-, two-, or three-dimensional (1-, 2-, or 3-D) space. These photonic crystals may be created by recording of periodic variations of amplitude and phase by the interference of coherent laser beams in holographic memory media. ${ }^{1-5}$ Recently we have analyzed and experimentally demonstrated that one can create a large number of general photonic crystals by holographic interferometry. ${ }^{4}$

A photonic crystal can change the propagation and transmission properties of light of wavelengths comparable with the nanometer dimensionality of a crystal lattice structure. ${ }^{6}$ When a light beam is passing through a photonic crystal and interacts with the crystal, interference between scattering waves may produce a photonic bandgap within which no propagating electromagnetic modes can exist.7,8 Based on

C. Chang is with the School of Electro-Optical Engineering, Yuan Ze University, Chungli 320, Taiwan, China. T.-M. Yan is with The Graduate Institute of Electro-Optical Engineering, Department of Electrical Engineering, National Taiwan University, No. 1 Sec. 4 Roosevelt Road, Taipei 106, Taiwan, China. H.-K. Liu (liuhuakuang@yahoo.com) is with the Department of Computer Science and Information Engineering, Tung Nan Institute of Technology, Shen-Keng, Taipei 122, Taiwan, China.

Received 12 July 2004; revised manuscript received 2 December 2004; accepted 3 December 2004.

0003-6935/05/132580-12 $\$ 15.00 / 0$

(C) 2005 Optical Society of America this property, device principles, including the guiding, bending, and filtering of waves, have been discovered. 9,10

One essential requirement in the fabrication of photonic crystals devices is the creation of defects. Creation of defects is often a challenge.

In this paper we present an analysis and an experimental demonstration of a double-exposure method for holographically creating photonic crystals with line defects. The idea is based on the principle of superposition of grating patterns of different spatial periods and thresholding the recording media. We use a specific symmetrical optical architecture that we recently reported ${ }^{4}$ to achieve the defective holographic photonic crystals. Section 2 is the theoretical analysis. In Section 3 we present an experimental demonstration of making holographic photonic crystals with and without line defects. A discussion and our conclusions are given in Section 4.

\section{Theoretical Analysis}

A. Creation of Holographic Photonic Crystals without Defects

As a helpful background, we first briefly review the fundamentals of the formation of a photonic crystal by means of the interference of four noncoplanar coherent laser beams ${ }^{4}$; then, based on the analysis, we present a novel idea for the creation of linear defects by superposition and application of a threshold to the recording medium of the photonic crystal. 
Assume that the complex electrical field of the $j$ th laser beam may be written as

$$
\overline{E_{j}}=E_{j} \exp \left[i\left(\overline{K_{j}} \cdot \bar{r}+\varphi_{o j}\right)\right] \overline{e_{j}}, \quad j=1,2,3,4,
$$

where $E_{j}, \bar{K}_{j}$, and $\varphi_{o j}$ denote the amplitude, the wave vector, and the initial phase of the jth laser beam, $\overline{e_{j}}$ is the unit vector of the wave's polarization, and $\bar{r}$ is an arbitrary vector. We further express the $j$ th wave vector as

$$
\overline{K_{j}}=\left(K_{j x}, K_{j y}, K_{j z}\right)=(2 \pi / \lambda)\left(s_{j}, t_{j}, u_{j}\right),
$$

where $s_{j}, t_{j}$, and $u_{j}$ are the direction cosines of the wave vector.

The intensity distribution of the interference field of the four noncoplanar beams can be written as

$$
\begin{aligned}
I= & \sum_{j} E_{j}^{2}+\sum_{i<j} 2 E_{i} E_{j} \cos \theta_{i j} \times \cos \left[\left(\overline{K_{i}}-\overline{K_{j}}\right) \cdot \bar{r}+\left(\varphi_{o i}\right.\right. \\
& \left.\left.-\varphi_{o j}\right)\right],
\end{aligned}
$$

where $\theta_{i j}$ is the angle between $\overline{e_{i}}$ and $\overline{e_{j}}$.

There are many ways to arrange the laser beams and to describe the laser beams by their corresponding wave vectors. We propose a practical way to arrange the interference of the four laser beams with the optical architecture block diagram shown in Fig. 1. This architecture is physically practical because the wave vectors can be measured and predetermined. As we show below, the architecture will facilitate the creation of linear defects.

\section{B. Method of Holographically Creating Line Defects in Photonic Crystals}

For device applications, quite often defects need to be built into photonic crystals. It has been found that defects can modify spontaneous emissions and form microcavities and waveguides. ${ }^{9-13}$ Various methods such as photolithography have been developed for the creation of defects in photonic crystals. However, for the first time as far as we know, we introduce the holographic creation of defective photonic crystals. The holographic method is feasible for the creation of $1-\mathrm{D}, 2-\mathrm{D}$, and 3-D defects. It is challenging to create the 3-D defects by any other methods.

The operation of the holographic method can be described beginning with Eq. (3), which shows that the intensity of the interference pattern is a sinusoidal function, and it is separable in spatial coordinates. Hence, for simplicity, we treat only one of the three dimensions.

The intensity distribution in the $x$ direction may be written as

$$
I(x)=B+A \cos \left(\frac{2 \pi}{T} x\right)
$$

where $B, A$, and $T$ are the parameters that represent the background intensity, the amplitude, and the spatial period, respectively, of the sinusoidal pattern.

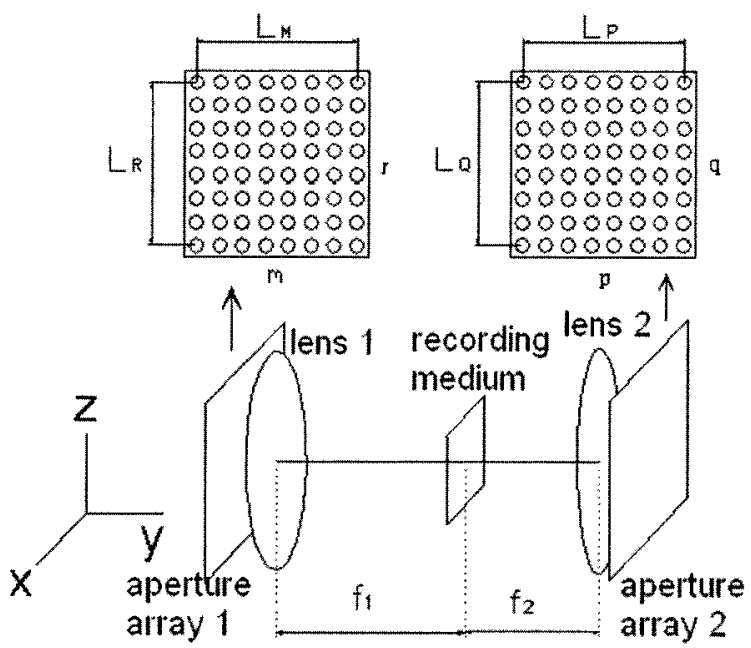

Fig. 1. Concept diagram of an optical architecture. In aperture array 1 the length and width are $L_{M}$ and $L_{R}$, respectively. The location of the hole opening is $(m, r)$. In aperture array 2 the length and width are $L_{P}$ and $L_{Q}$. The location of the hole opening is $(p, q)$. The focal lengths of lens 1 and lens 2 are $f_{1}$ and $f_{2}$, respectively.

We assume that the holographic medium is first properly double exposed ${ }^{14,15}$ and then developed to high contrast with a threshold. In the doubleexposure process we sequentially make two planewave exposures of the holographic recording medium. The total intensity received by the medium may be written as

$$
\begin{aligned}
I_{t}(x) & =B_{1}+A_{1} \cos \left(\frac{2 \pi}{T_{1}} x\right)+B_{2}+A_{2} \cos \left(\frac{2 \pi}{T_{2}} x\right) \\
& =C+A_{1} \cos \left(\frac{2 \pi}{T_{1}} x\right)+A_{2} \cos \left(\frac{2 \pi}{T_{2}} x\right),
\end{aligned}
$$

where $C=B_{1}+B_{2}$ denotes the combined background intensity of the two exposures. If the recording medium has infinite or extremely high contrast, such as that provided by a high-gamma photographic emulsion, then we may assume that there is a threshold value of the exposure, $I_{\text {th }}$. The gamma of the emulsion may be controllable by the type of medium and developer used and the time and temperature of development.

Based on the threshold principle, ${ }^{16,17}$ which is similar to that used in nonlinear image processing and halftone graphic arts printing, a variety of defects may be added to the photonic crystals. We show the principle of operation through a computer simulation as follows.

We assume that the threshold of the recording medium is a constant, $I_{\text {th }}$. For concept demonstration and simplicity in simulation, we let $A_{1}=1, T_{1}=1$, $A_{2}=A$, and $T_{2}=T$, and then rewrite Eq. (5) as

$$
I_{t}(x)=C+\cos (2 \pi \mathrm{x})+A \cos \left(\frac{2 \pi}{T} x\right)
$$


Table 1. Photonic Crystal Samples with Fixed Relative Period $T$ and Various Relative Amplitudes A and Thresholds $I_{\text {th }}$

\begin{tabular}{|c|c|c|c|c|c|c|c|c|c|c|}
\hline \multirow[b]{2}{*}{ Parameter } & \multicolumn{10}{|c|}{ Sample } \\
\hline & $\mathrm{a}$ & $\mathrm{b}$ & $\mathrm{c}$ & $d$ & $\mathrm{e}$ & $\mathrm{f}$ & $\mathrm{g}$ & $\mathrm{h}$ & i & $\mathrm{j}$ \\
\hline$T$ & 20 & 20 & 20 & 20 & 20 & 20 & 20 & 20 & 20 & 20 \\
\hline$A$ & 0.1 & 0.1 & 0.2 & 0.2 & 0.3 & 0.3 & 0.4 & 0.5 & 0.5 & 0.5 \\
\hline$I_{\text {th }}$ & 2 & 2.005 & 2 & 2.01 & 2 & 2.015 & 2 & 2.02 & 2 & 2.025 \\
\hline
\end{tabular}

Because the total background intensity $(C)$ and the threshold $\left(I_{\mathrm{th}}\right)$ have the same effect in the simulation, for simplicity we consider only the threshold value. The following examples will show how relative period $(T)$, relative amplitude $(A)$, and threshold $\left(I_{\text {th }}\right)$ can be used to control the creation of defects.

\section{Example 1. Line Defect Created with Fixed Relative Period T in One-Dimensional Holographic Photonic Crystals}

In Table 1 we fix the relative period by assigning it a value of 20 and classify the samples of the photonic crystals (a)-(j) by varying the amplitude and threshold values. The corresponding transmittance or, equivalently, the dielectric constant distributions of sample (a)-(j), are shown in Fig. 2.

From Fig. 2 we can see a unique 1-D grating structure. The broad spaces in dielectric constant plots centered at positions -10 and +10 represent the defects. Comparing Figs. 2(a)-2(j), we can see that the width of the defects is controllable by the threshold. The width of each fine grating element varies according to the relative amplitude and threshold. The fine grating elements of the same width also form a periodic structure of the same period. This is a distinctive feature of the defective holographic photonic crystals.

In addition to the method of fixing the relative period, the line defects in the holographic photonic crystals can also be created with fixed relative amplitude (A). For instance, in Table 2 we fix relative amplitude $A$ by assigning it the value 0.1 and we classify the samples of photonic crystals (a)-(e) by varying the relative period and threshold. The corresponding transmittance, or, equivalently, the dielectric constant distributions of samples (a)-(e) are similar to those shown in Fig. 2. The relative amplitude controls the period of the defects. The threshold value affects the widths of the fine gratings and defects the same way as shown in Example 1. The fine grating elements of the same width form a periodic structure by themselves and are of the same period. One constraint in this case is that the relative period $(T)$ must be an integer; otherwise the periodicity will be destroyed.

We have analyzed the holographic method of creating 1-D photonic crystal with defects. In principle, the method is applicable to 2-D and 3-D photonic crystals. To keep the demonstration simple and show the generality of the approach, we present a computer simulation example in two-dimensions below.

\section{Example 2. Two-Dimensional Photonic Crystals} with Line Defects

In the 2-D case we can use four specific laser beams to generate a square interference pattern. Then we can use two beams to successively expose the recording medium to generate a 1-D line pattern. We let the relative period and amplitude of the line and square patterns be $T$ and $A$, respectively, which are similar to those defined in 1-D cases. The line defects will be created and will vary periodically as expected.

We may classify the defects into air defects and dielectric defects, depending on the method of formation of the defects. An air defect becomes a vacuum defect if the device is used in vacuum. To create an air defect we let $T=8, A=0.1$, and $I_{\mathrm{th}}=0.2$. The corresponding transmittance functions or, equivalently, dielectric constant distributions, are shown in Fig. 3(a). For a dielectric defect we let $T=8, A=1.1$, and $I_{\text {th }}$ $=1.1$, and the results are shown in Fig. 3(b).

The defects created in both of these two cases may be treated and used as multichannel waveguides. As we let $T=8$ in these examples, the spacing between any two nearest-neighbor channels or between the line defects is separated by eight lattice constants of the photonic crystal. If, instead, we want to create only one channel, we can increase $T$ appropriately.

The examples above show that the photonic crystals are composed of fine gratings of different widths that are controlled by the threshold of the recording medium and the amplitude of the beams. As we use only two sinusoidal spatial functions to create the defect structure, the sizes of the elements of the fine gratings are different. A more general defective photonic crystal can in principle normally be synthesized by a Fourier series of sinusoidal basis functions. But it is experimentally difficult, if not impossible, to achieve many of the arbitrary defects. If we use a few more functions to approximately synthesize, for example, the square function, the grating shapes can only be made closer to the true square function.

\section{Experimental Demonstration of Making Holographic Photonic Crystals with and without Line Defects}

\section{A. Experimental System}

The optical system that we use to create the holographic photonic crystals ${ }^{4}$ with and without defects 
(a)
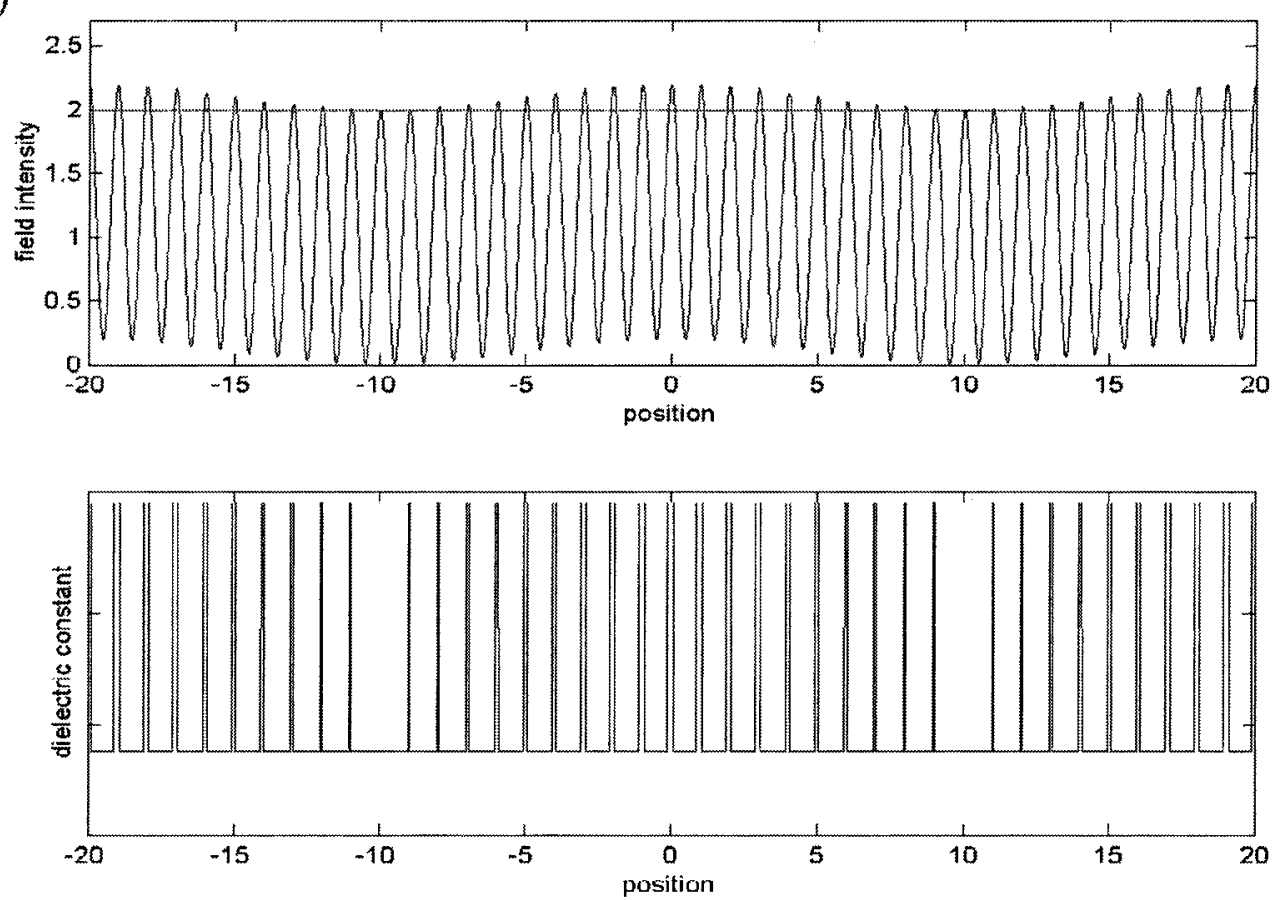

(b)
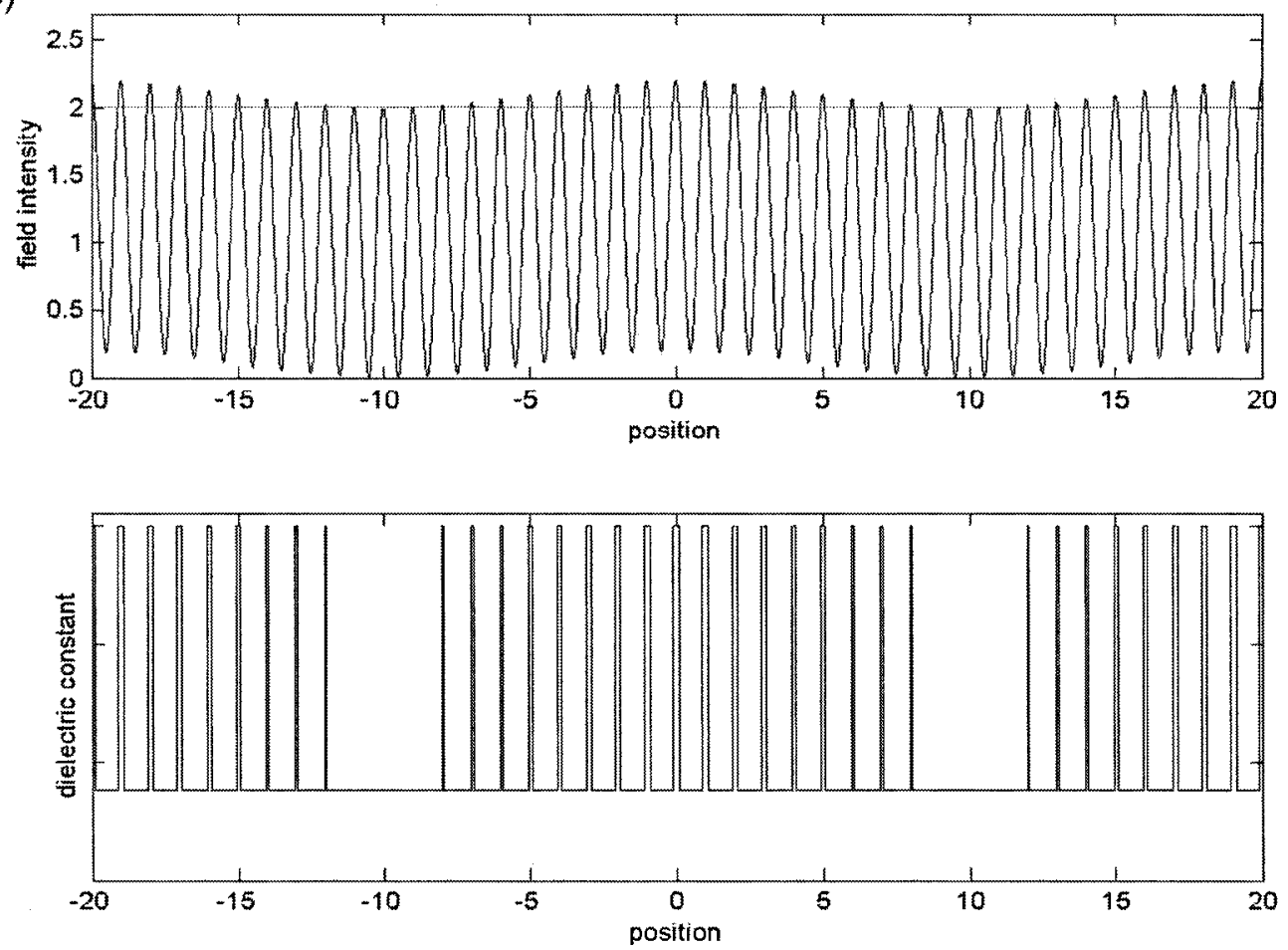

Fig. 2. Continues on next page.

is shown in Fig. 4. A 10-mW maximum power laser diode of $650-\mathrm{nm}$ wavelength is used as the light source for the system, and its power level is adjustable. First we collimate the laser beam by passing it through a spatial filter and then split it into two laser beams. Each of the two beams passes through a dif- fractive optical element, which is an $8 \times 8$ array generator. Sixty-four nearly uniform laser beams are produced and propagate from opposite sides that can be focused to meet at the holographic recording medium. Before the $8 \times 8$ laser beams from each side meet at the medium, an $8 \times 8$ aperture array is used 

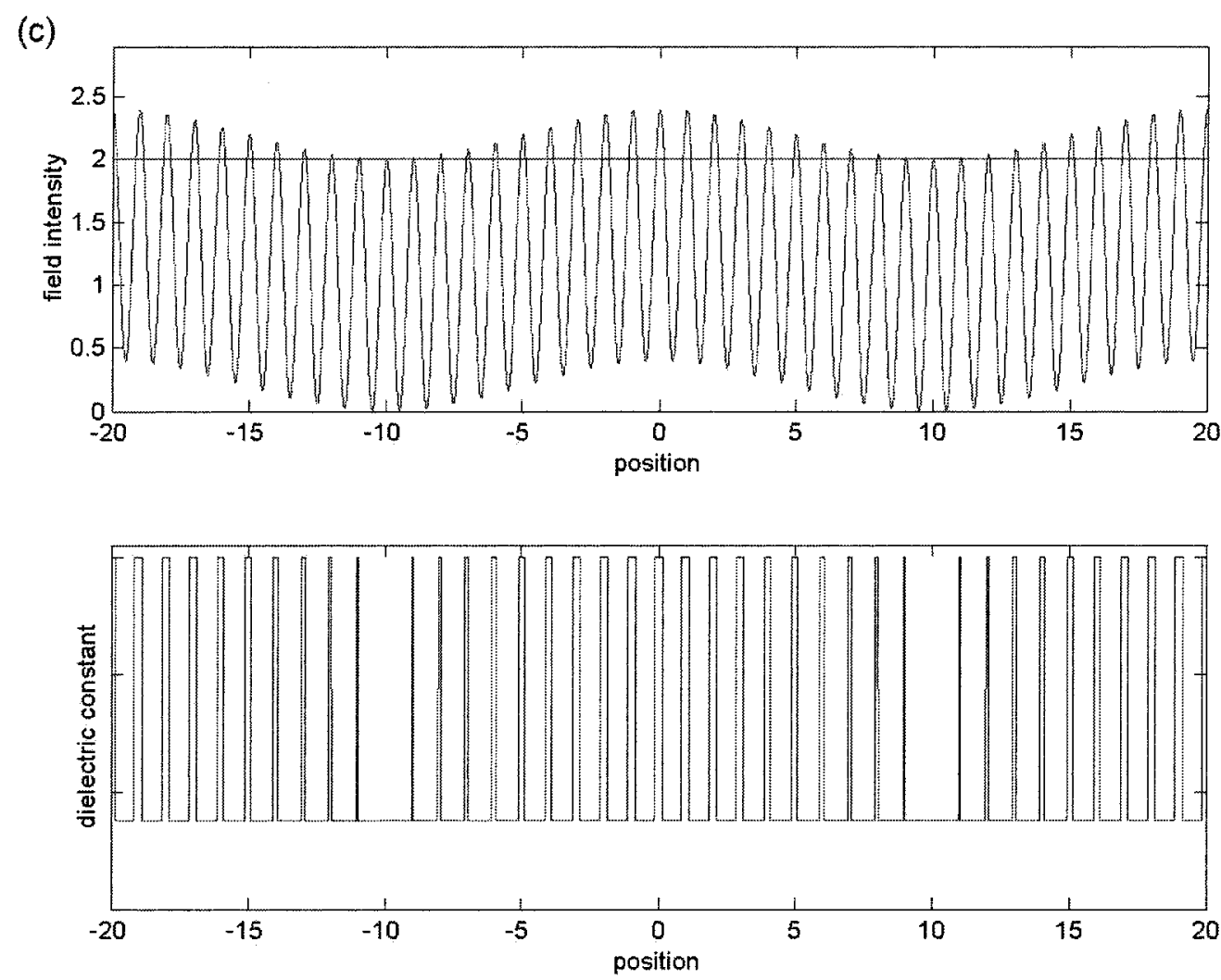

(d)
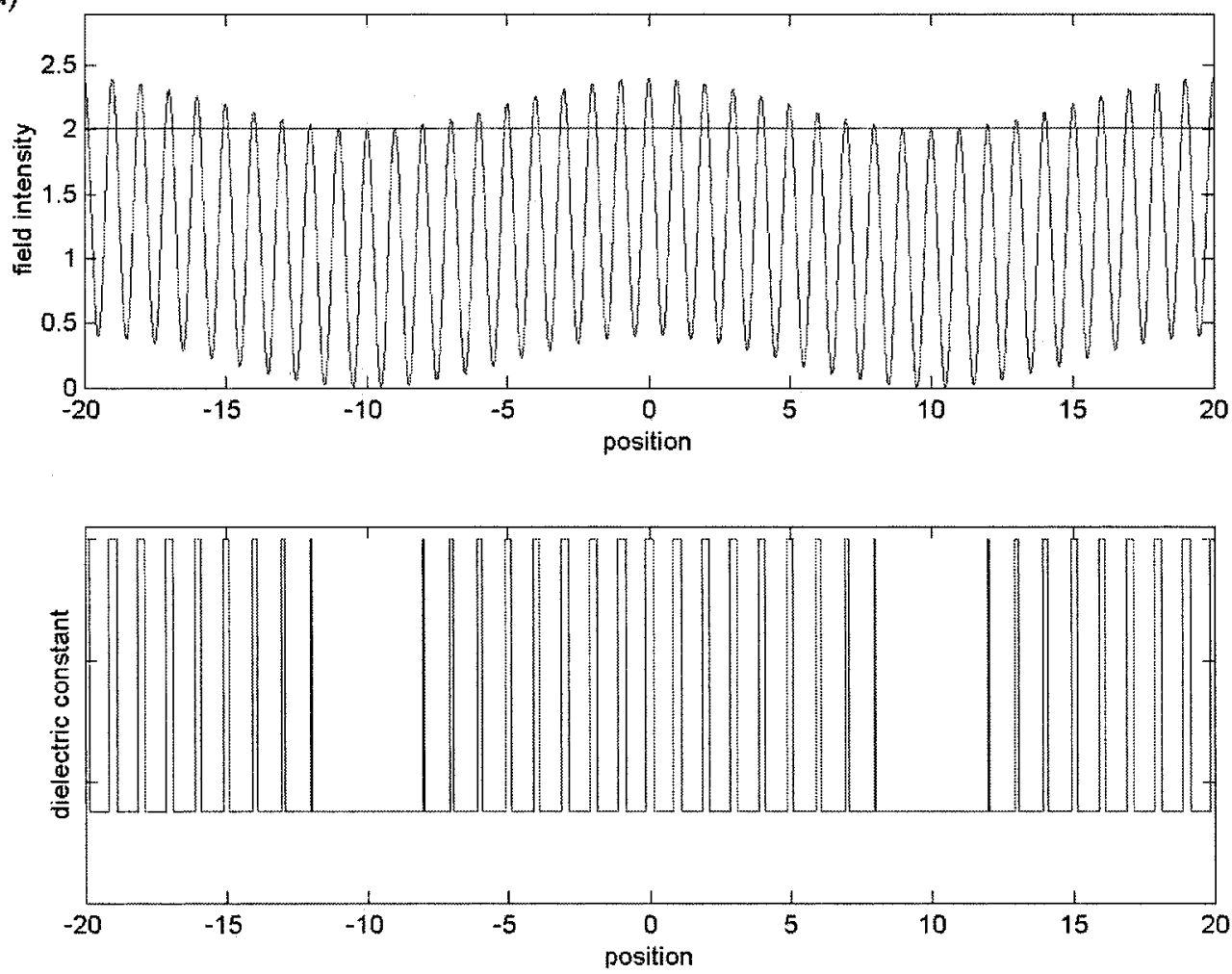

Fig. 2. Continues on next page.

to select the beams by opening or closing each of the apertures. The wave vectors of the selected laser beams are determinable by the positions at which the open apertures are chosen. The selected laser beams 

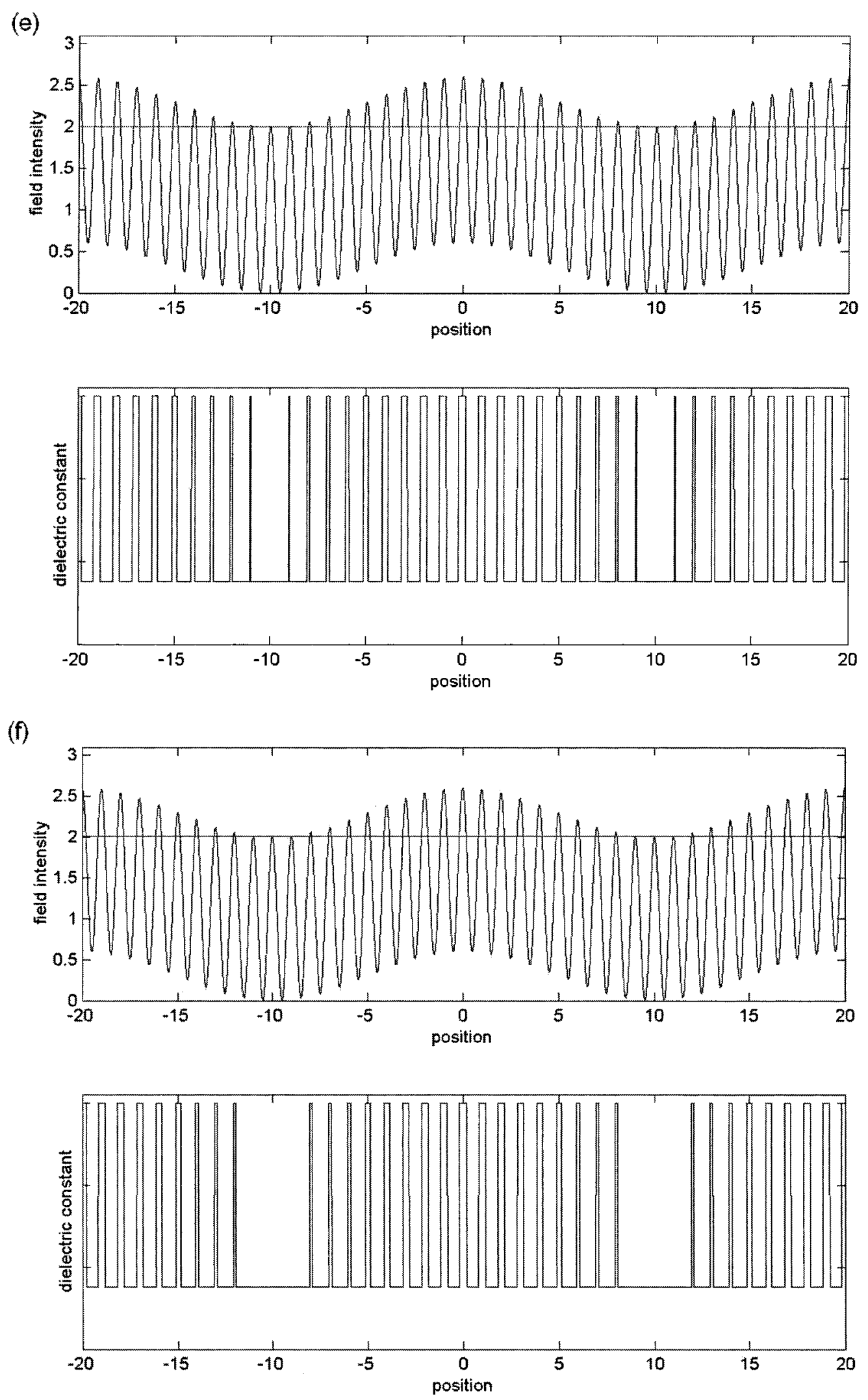

Fig. 2. Continues on next page.

from each side are focused at the recording medium by a convex lens to create the photonic crystal.
The holographic recording medium that we used is silver halide emulsion PFG-01 (Stavich Company, 
(g)
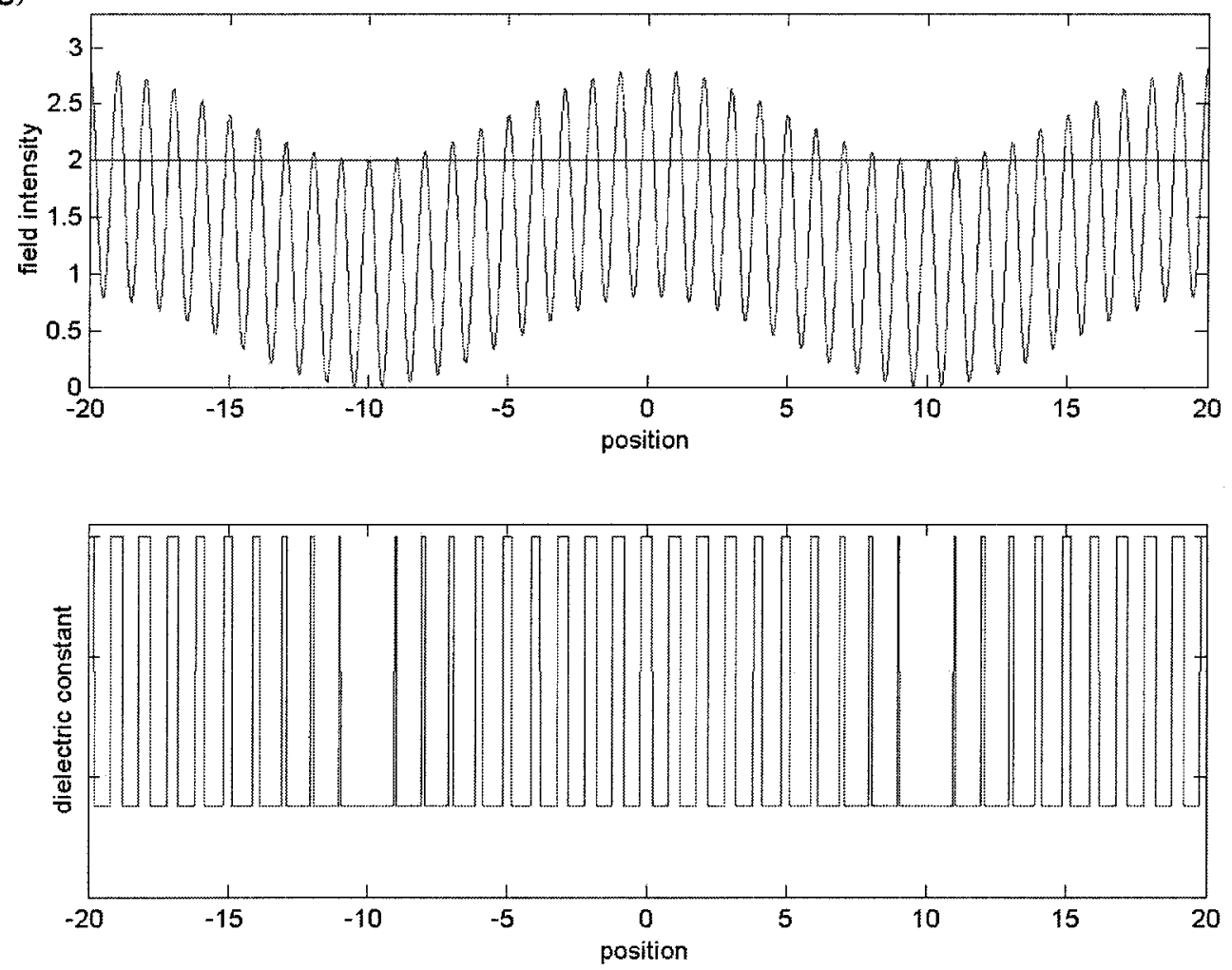

(h)
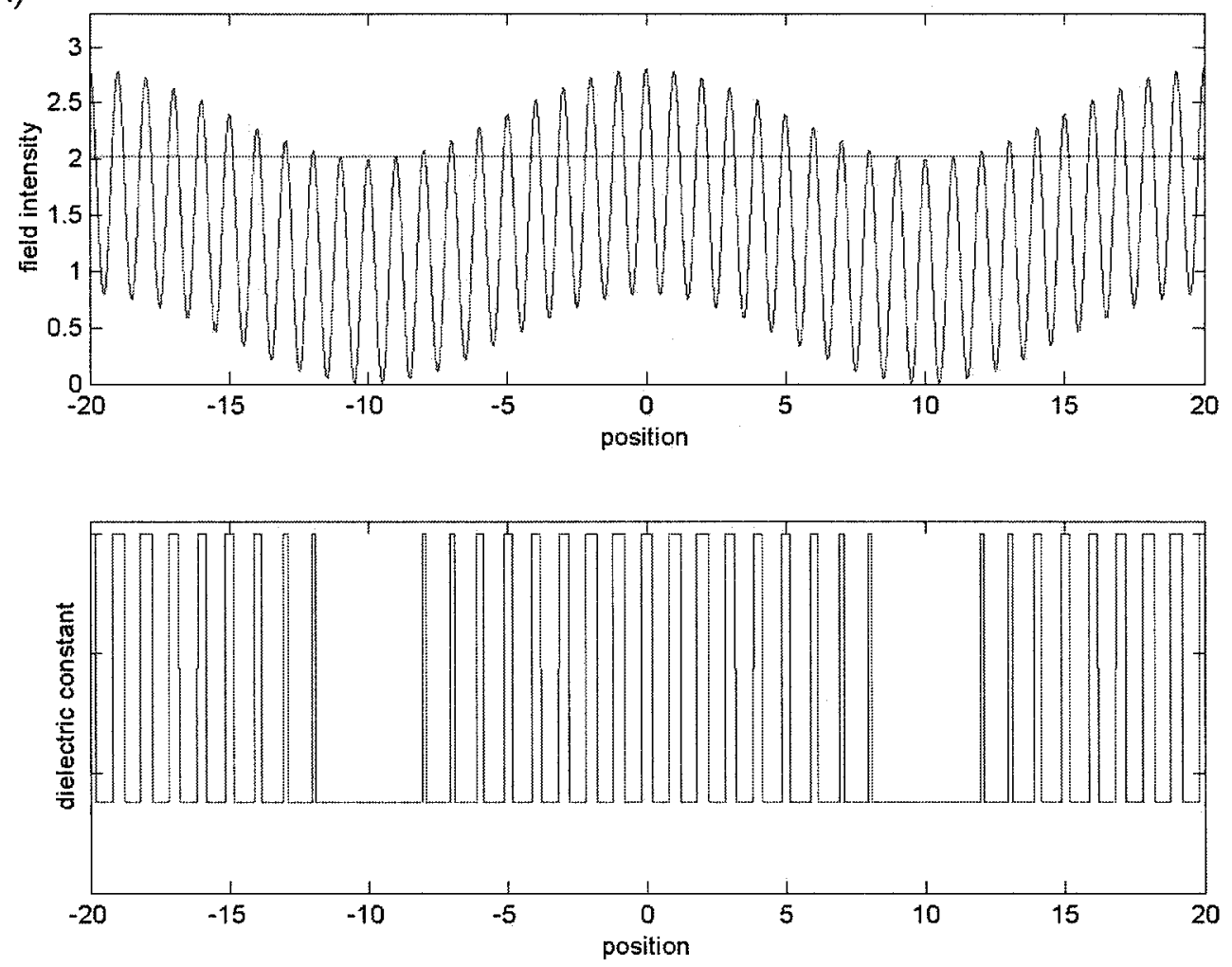

Fig. 2. Continues on next page.

Ltd.), with a resolution higher than 3000 lines/mm. The emulsion is made from fine-grain silver halide crystals suspended in gelatin. After development and fixing, the exposed regions are left with silver parti- 

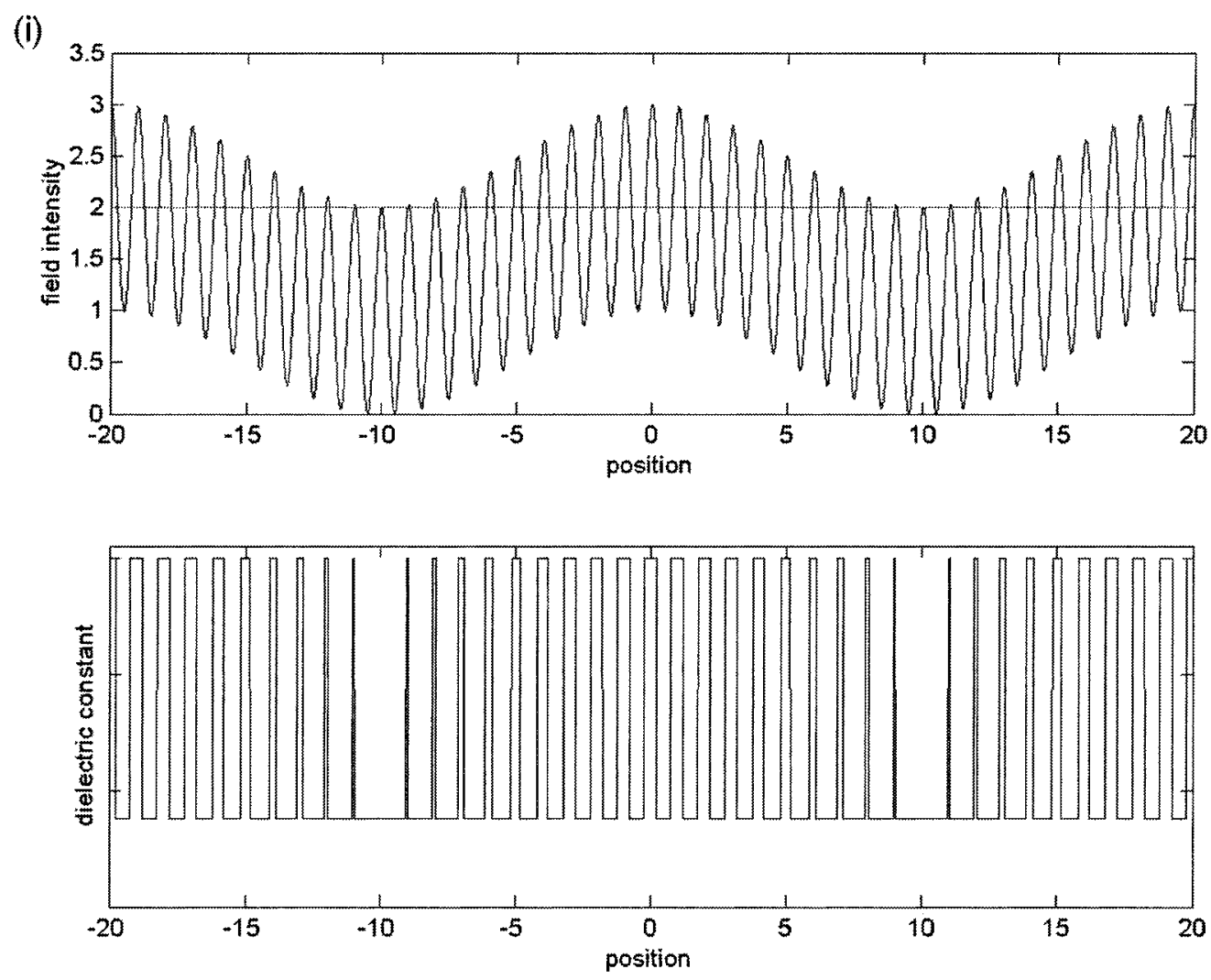

(j)
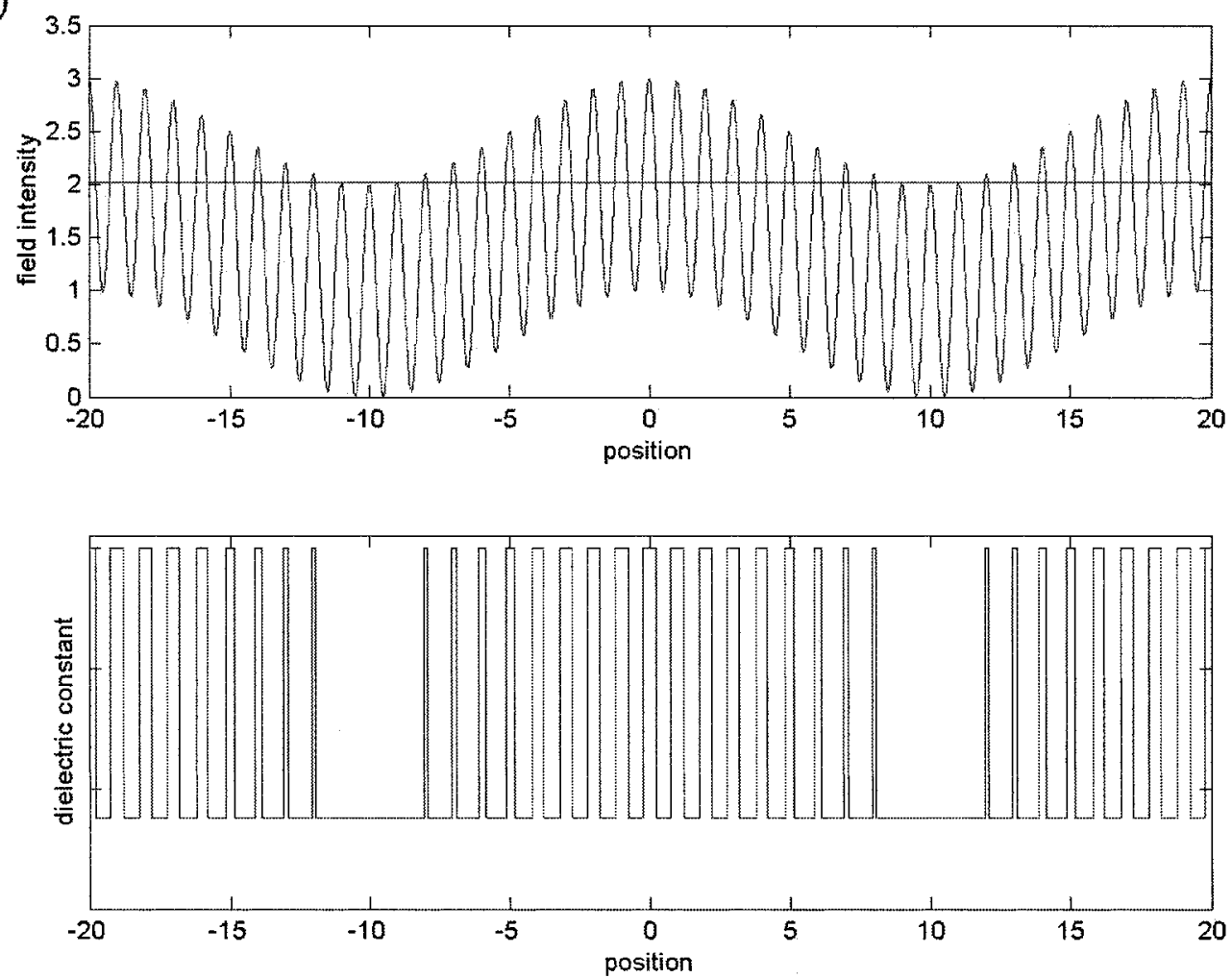

Fig. 2. Intensity and dielectric constant distribution with fixed relative period T. (a)-(j) Photonic crystal samples a-j described in Example 1 and Table 1. The horizontal line in the field intensity plot denotes the threshold. 
Table 2. Photonic Crystal Samples Classified with Fixed Relative Amplitude A and Varying Relative Period $T$ and Threshold $I_{\text {th }}$

\begin{tabular}{cccccl}
\hline & \multicolumn{5}{c}{ Sample } \\
\cline { 2 - 6 } Parameter & $\mathrm{a}$ & $\mathrm{b}$ & $\mathrm{c}$ & $\mathrm{d}$ & $\mathrm{e}$ \\
\hline$T$ & 20 & 15 & 10 & 8 & 5 \\
$A$ & 0.1 & 0.1 & 0.1 & 0.1 & 0.1 \\
$I_{\text {th }}$ & 2 & 2.0025 & 2 & 2 & 2.02 \\
\hline
\end{tabular}

cles in gelatin whose density depends on exposure, and the unexposed regions are washed out. The average dielectric constant is 2.686 (measured by the Stavich Company at a wavelength of $640 \mathrm{~nm}$ ). This dielectric constant is larger than that of the popularly used resin SU8 for making holographic photonic crys-

(a)

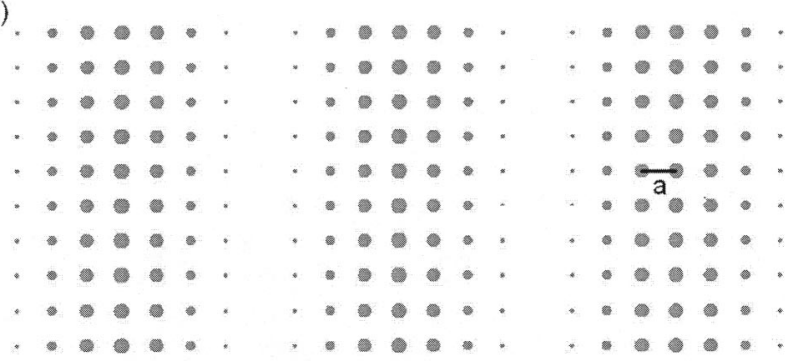

(b)

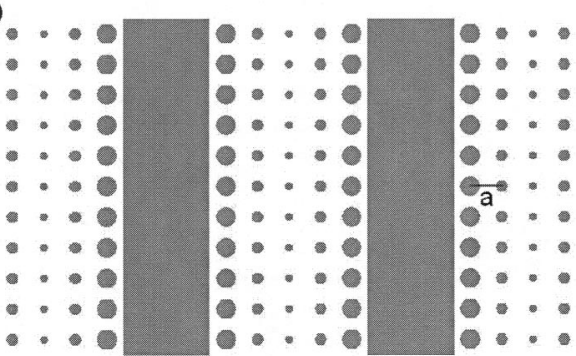

Fig. 3. Dielectric constant distribution of Example 2: (a) air defect, (b) dielectric defect.

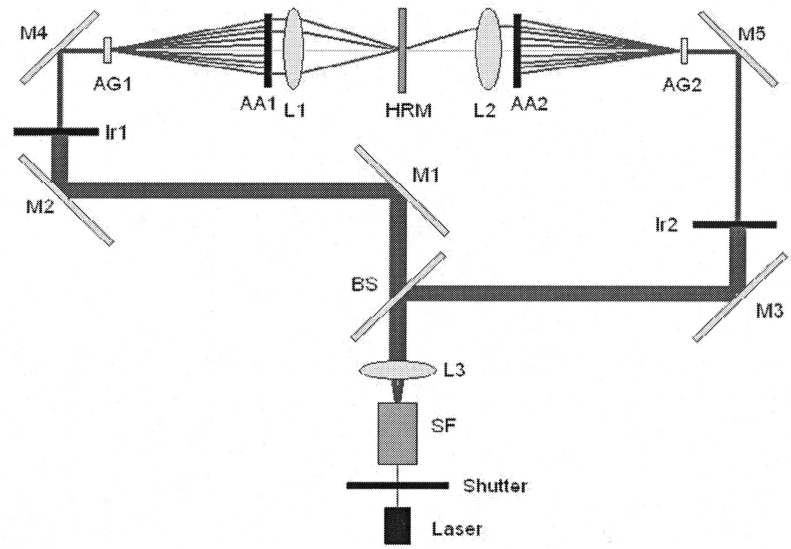

Fig. 4. Experimental setup for the conceptual optical architecture shown in Fig. 1: M1-M5, mirrors; BS, beam splitter; AG1, AG2, array generators; AA1, A2, aperture arrays; HRM, holographic recording medium; L1-L3, lenses; SF, spatial filter; Ir1, Ir2, irises. tals $(\varepsilon=2.56) .^{18-22}$ In what follows, we present the results of our experiments with photonic crystals and with photonic crystals with line defects. The 3 -D photonic crystal created by this method has been described elsewhere. ${ }^{4}$

\section{B. Experimental Results}

\section{Two-Dimensional Holographic Photonic Crystals}

For creating the 2-D photonic crystals we found that three beams from one side only in the optical system are sufficient. Referring to the previous theoretical discussion, ${ }^{4}$ we selected the open apertures at $(m, r)=(3,3),(6,6),(3,6)$ in the aperture array from one side of the recording medium; a lens with a focal length of $10 \mathrm{~cm}$ is used for focusing of the three beams. The distance between the lens and the recording medium is $f_{1}=11.8 \mathrm{~cm}$. The power is $260 \mathrm{lux}$. The selected open apertures enable us to measure and determine the three wave vectors as

$$
\begin{aligned}
& \overline{K_{1}}=(0.0560,0.9969,-0.0560), \\
& \overline{K_{2}}=(-0.0560,0.9969,0.0560), \\
& \overline{K_{3}}=(0.0560,0.9969,0.0560),
\end{aligned}
$$

from which we calculate two primitive lattice constants in micrometer units:

$$
\begin{aligned}
& \bar{A}=(5.804,0,0), \\
& \bar{B}=(0,0,5.804) .
\end{aligned}
$$

With the above setup, a 2-D photonic crystal is created. The crystal structure is examined under an optical microscope. Three $1000 \times$ microscope images of the sample in the $x-z$ plane are shown in Fig. 5. For Fig. 5(a) the exposure time was $0.5 \mathrm{~s}$. The measured lengths of the primitive lattice constants $\bar{A}$ and $\bar{B}$ are 5.770 and $5.229 \mu \mathrm{m}$, respectively. The ratio of radius to lattice constant $(r / a)$ is $\sim 0.355$. For Fig. $5(b)$ the exposure time was $0.5 \mathrm{~s}$. The measured lengths of primitive lattice constants $\bar{A}$ and $\bar{B}$ are 5.714 and $5.294 \mu \mathrm{m}$, respectively. The ratio of radius to lattice constant is $\sim 0.287$. For Fig. 5(c) the exposure time was $0.125 \mathrm{~s}$. The measured lengths of primitive lattice constants $\bar{A}$ and $\bar{B}$ are 5.826 and $5.177 \mu \mathrm{m}$, respectively. The ratio of radius to lattice constant is $\sim 0.260$. The differences in Figs. 5(a)-5(c) are that the longer the exposure time, the bigger the interference pattern or radius-to-lattice constant ratio. Both the ratio and the lattice constant exhibit calculated results of the same order, with some errors.

\section{Creating Line Defects Holographically in}

Holographic Photonic Crystals

We have used the double-exposure method described in Subsection 2.B to create line defects experimen- 
(a)

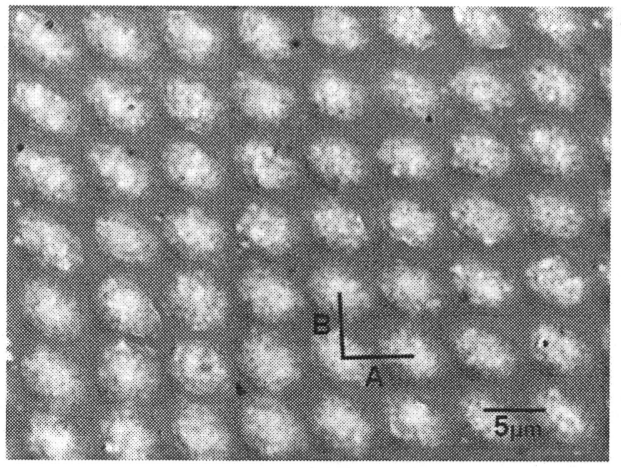

(b)

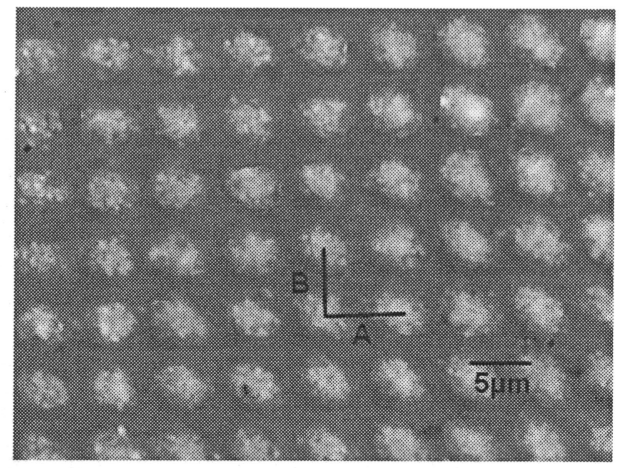

(c)

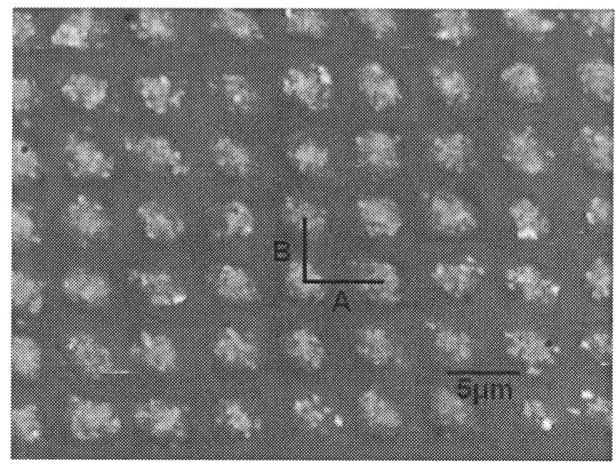

Fig. 5. $1000 \times$ microscope image in the $x-z$ plane of a 2-D photonic crystal. (a) Exposure time, is $0.5 \mathrm{~s} . A=5.770 \mu \mathrm{m}, B=5.229 \mu \mathrm{m}$. (b) Exposure time, $0.25 \mathrm{~s}$. $A=5.714 \mu \mathrm{m}, B=5.294 \mu \mathrm{m}$. (c) Exposure time, $0.125 \mathrm{~s} . A=5.826 \mu \mathrm{m}, B=5.177 \mu \mathrm{m}$. Notice that we call the $y$ axis the axis of propagation, not the $z$ axis as is conventionally done.

tally in the holographic photonic crystals. We can make photonic crystals with different defects by adjusting the wavelength, open apertures, focal length, exposure time, and developing time, etc. An example follows.

For the first exposure we opened the apertures at $(m, n)=(1,1),(1,8),(8,8),(8,1)$ in the aperture array. The distance between the lens and the recording medium was $f_{1}=12 \mathrm{~cm}$. For the second exposure, the open apertures were at $(m, n)=(4,4),(5,4)$ in the aperture array. The distance between the lens and the recording medium was kept at $f_{1}=12 \mathrm{~cm}$. The power level of the laser was not changed (437 lux) for both exposures. The selected open apertures determined the primitive lattice constants to be

$$
\begin{array}{cl}
\text { First exposure: } & \bar{A}=(2.291,0,0) \mu \mathrm{m}, \\
& \bar{B}=(0,0,2.291) \mu \mathrm{m}, \\
\text { Second exposure: } & \bar{D}=(0,0,15.720) \mu \mathrm{m},
\end{array}
$$

where $t=0.25 \mathrm{~s}$. A 2 -D photonic crystal with line dielectric defects is formed, as simulated in Fig. 3(b). A $500 \times$ microphotograph of the photonic crystal created in this manner is shown in Fig. 6. The measured lengths of primitive lattice constants $\bar{A}, \bar{B}$, and $\bar{D}$ are $2.531,2.353$, and $16.255 \mu \mathrm{m}$, respectively, which are close to the calculated values.

The defects made by the dielectric material are similar to those in the traditional waveguide. The dominant guiding effect is due to total internal reflection rather than to the photonic bandgap.

For an air defect the first exposure was made with apertures open at $(m, n)=(2,2),(2,7),(7,7),(7,2)$ in the array. The distance between the lens and the

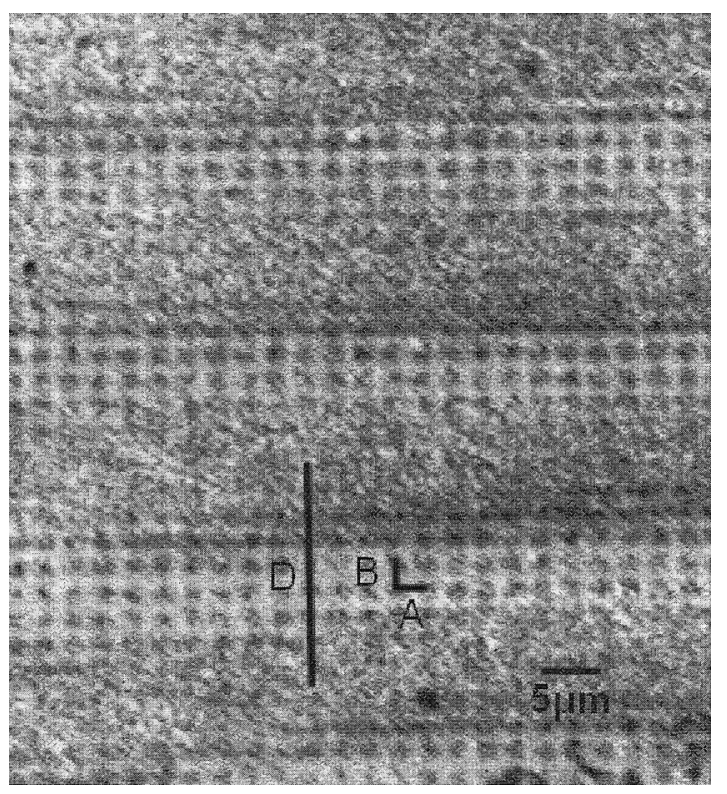

Fig. 6. Microscopic image of a 2-D photonic crystal with line dielectric defects. The measured lengths of $\bar{A}, \bar{B}$, and $\bar{D}$ are 2.531, 2.353 , and $16.255 \mu \mathrm{m}$, respectively. 
recording medium was $f_{1}=4.7 \mathrm{~cm}$. For the second exposure the open apertures were at $(m, n)$ $=(4,4),(5,4)$ in the aperture array. The distance between the lens and the recording medium remained $f_{1}=5.5 \mathrm{~cm}$. The power level of the laser (367 lux) was not changed for both exposures. The selected open apertures enabled us to determine the primitive lattice constants as follows:

$$
\text { First exposure: } \begin{aligned}
\bar{A} & =(1.314,0,0) \mu \mathrm{m}, \\
\bar{B} & =(0,0,1.314) \mu \mathrm{m}, t=0.03 \mathrm{~s} ;
\end{aligned}
$$

Second exposure: $\bar{D}=(0,0,6.563) \mu \mathrm{m}, t=0.004 \mathrm{~s}$.

We found that a 2-D photonic crystal with line air defects was formed as simulated in Fig. 3(a). The measured lengths of primitive lattice constants $\bar{A}, \bar{B}$, and $\bar{D}$ are $1.386,1.307$, and $6.797 \mu \mathrm{m}$, respectively, which are also close to the calculated values. Because the spacing between the line defects is only approximately five lattice constants, the bandgap guiding effect is not clear.

\section{Discussion and Conclusions}

We have presented and demonstrated experimentally a method of creating holographic photonic crystals with and without line defects in a symmetrical optical system. The line defects were created by double exposure and thresholding of the recording medium. In the examples presented, some assumptions in the analysis may cause discrepancies between the measured and calculated results. For instance, we assume that the phase difference between any two beams is zero in Eq. (3). Actually, the phase difference may not be zero because the beams pass through the beam splitter and the diffractive optical element (array generator). But, as shown by the experimental results, the effect is not serious, probably because of the symmetry of our optical system. Besides, errors in the optical microscopy measurement, in experimental setup, and in the contrast of the recording medium may also contribute to inaccuracies. Because of the high resolution of the silver halide emulsion (3000-5000 lines $/ \mathrm{mm}$ ) used in recording the photonic crystals, the sensitivity and gamma or contrast is naturally low. Hence, although we have used special techniques, such as extending the development time and increasing the temperature, to enhance the gamma, there was still not a clear-cut sharp threshold value for the hard clipping effect to be apparent. That is the reason that the photograph of the line defect in Fig. 6 is fuzzy. The fuzziness will cause the waveguide not to function and will generate noise in the processed signal. Obviously the silver halide film is not an ideal material for creation of photonic crystals. Other recording materials of high resolution, high sensitivity, and high contrast with sharp threshold are needed for the method.

The holographic photonic crystals with defects cre- ated with the new method are unique because their unit cells are of different sizes.

Based on our band structure calculations not given in this paper, holographic photonic crystals do not have any bandgap if the recording medium used is silver halide and the resultant dielectric constant is low. If we need to design devices such as waveguides and filters, large dielectric constant materials have to be used. In that case we may choose another recording medium with high index or use our holographic photonic crystal as a template for electrodeposition of a large dielectric constant material. The feasibility of making an inverse replica to obtain a photonic crystal with high index contrast has been reported in the literature. ${ }^{22}$

Finally, in our optical architecture the important factors for making photonic crystals with smaller lattice constants are the wavelength of the light source and the numerical aperture of the lens. Because of the limited components available in our laboratory when the research was conducted, the smallest lattice constant of the created photonic crystal was $\sim 0.8 \mu \mathrm{m}$, and its bandgap did not reach the visible spectrum. However, theoretical analysis and experimental results have demonstrated the feasibility of the method.

For future research we suggest that a variety of recording materials, light sources, and exposure steps be used to create a variety of photonic crystals. The exploration of high-contrast recording media will greatly improve the ability to make line defects in photonic crystals.

This research has been supported by the Republic of China's National Research Council grants 92-2215E-002-026 and 92-2215-E-236-1 and -2. We appreciate helpful technical discussions with Yean-Woei Kiang, Lon A. Wang, and Jer-Ren Yang. Hua-Kuang Liu is thankful for the support of the Center for Engineering Science Advanced Research, Oak Ridge National Laboratory, Oak Ridge, Tennessee where he has been working as a Distinguished Visiting Scientist.

\section{References}

1. M. Campbell, D. N. Sharp, M. T. Harrison, R. G. Denning, and A. J. Turberfield, "Fabrication of photonic crystals for the visible spectrum by holographic lithography," Nature 404, 53-56 (2000).

2. T. Kondo, S. Matsuo, S. Juodkazis, and H. Misawa, "Femtosecond laser interference technique with diffractive beam splitter for fabrication of three-dimensional photonic crystals," Appl. Phys. Lett. 79, 725-727 (2001).

3. L. Z. Cai, X. L. Yang, and Y. R. Wang, "All fourteen Bravais lattices can be formed by interference of four noncoplanar beams," Opt. Lett. 27, 900-902 (2002).

4. T. M. Yan and H.-K. Liu, "Holographic creation of photonic crystals," Appl. Opt. 43, 4376-4384 (2004).

5. X. L. Yang, L. Z. Cai, Q. Liu, and H. K. Liu, "Theoretical bandgap modeling of two-dimensional square photonic crystals fabricated by the interference of three noncoplanar laser beams," J. Opt. Soc. Am. B 21, 1699-1702 (2004).

6. E. Yablonovitch, "Inhibited spontaneous emission in solid- 
state physics and electronics," Phys. Rev. Lett. 58, 2059-2062 (1987).

7. J. D. Joannopoulos, P. R. Villeneuve, and S. Fan, "Photonic crystals: putting a new twist on light," Nature 386, 143-149 (1997).

8. T. F. Krauss and R. M. De La Rue, "Photonic crystals in the optical regime-past, present and future," Prog. Quantum Electron. 23, 51-96 (1999).

9. S. Y. Lin and E. Chow, "Experimental demonstration of guiding and bending of electromagnetic waves in a photonic crystal," Science 282, 274-276 (1998).

10. J. S. Foresi, P. R. Villeneuve, J. Ferrera, E. R. Thoen, G. Steinmeyer, S. Fan, J. D. Joannopoulos, L. C. Kimerling, H. I. Smith, and E. P. Ippen, "Photonic-bandgap microcavities in optical waveguides," Nature 390, 143-145 (1997).

11. C. Manolatou, M. J. Khan, S. Fan, P. R. Villeneuve, H. A. Haus, and J. D. Joannopoulos, "Coupling of modes analysis of resonant channel add-drop filters," IEEE J. Quantum Electron. 35, 1322-1331 (1999).

12. B. D'Urso, O. Painter, J. O'Brien, T. Tombrello, A. Yariv, and A. Scherer, "Modal reflectivity in finite-depth two-dimensional photonic-crystal microcavities," J. Opt. Soc. Am. B 15, 11551159 (1998).

13. M. Loncar, D. Nedeljkovic, T. Doll, J. Vuckovic, A. Scherer, and T. Pearsall, "Waveguiding in planar photonic crystals," Appl. Phys. Lett. 77, 1937-1939 (2000).

14. S. Shoji and S. Kawata, "Photofabrication of three-dimensional photonic crystals by multibeam laser interference into a photopolymerizable resin," Appl. Phys. Lett. 76, 2668-2670 (2000).

15. S. Shoji, H. B. Sun, and S. Kawata, "Photofabrication of wood- pile three-dimensional photonic crystals using four-beam laser interference," Appl. Phys. Lett. 83, 608-610 (2003).

16. H. K. Liu, J. W. Goodman, and J. L.-H. Chan, "Equidensitometry by coherent optical filtering," Appl. Opt. 15, 2394-2400 (1976).

17. H.-K. Liu, "Coherent optical analog-to-digital conversion using a single halftone photograph," Appl. Opt. 17, 2181-2185 (1978).

18. M. Campbell, D. N. Sharp, M. T. Harrison, R. G. Denning, and A. J. Turberfield, "Fabrication of photonic crystals for the visible spectrum by holographic lithography," Nature 404, 53-56 (2000).

19. Yu. V. Miklyaev, D. C. Meisel, A. Blanco, G. von Freymann, K. Busch, W. Koch, C. Enkrich, M. Deubel, and M. Wegener, "Three-dimensional face-centered-cubic photonic crystal templates by laser holography: fabrication, optical characterization, and band-structure calculations," Appl. Phys. Lett. 82, 1284-1286 (2003).

20. I. B. Divliansky, A. Shishido, I. C. Khoo, T. S. Mayer, D. Pena, S. Nishimura, C. D. Keating, and T. E. Mallouk, "Fabrication of two-dimensional photonic crystals using interference lithography and electrodeposition of CdSe," Appl. Phys. Lett. 79, 3392-3394 (2001).

21. I. B. Divliansky, T. S. Mayer, K. S. Holliday, and V. H. Crespi, "Fabrication of three-dimensional polymer photonic crystal structures using single diffraction element interference lithography," Appl. Phys. Lett. 82, 1667-1669 (2003).

22. X. Wang, J. F. Xu, H. M. Su, Z. H. Zeng, Y. L. Chen, H. Z. Wang, Y. K. Pang, and W. Y. Tam, "Three-dimensional photonic crystals fabricated by visible light holographic lithography," Appl. Phys. Lett. 82, 2212-2214 (2003). 